\title{
TRACE INEQUALITIES FOR MULTIPLE PRODUCTS OF TWO MATRICES
}

\author{
TSUyoshi ANDO, Fumio Hiai AND KAZUyoshi OKubo
}

\begin{abstract}
Trace inequalities for multiple products of powers of two matrices are discussed via the method of $\log$ majorization. For instance, the trace inequality $\left|\operatorname{Tr}\left(A^{p_{1}} B^{q_{1}} A^{p_{2}} B^{q_{2}} \cdots A^{p_{K}} B^{q_{K}}\right)\right| \leqslant$ $\operatorname{Tr}(A B)$ is obtained for positive semidefinite matrices $A, B$ and $p_{i}, q_{i} \geqslant 0$ with $p_{1}+\cdots+p_{K}=$ $q_{1}+\cdots+q_{K}=1$ under some additional condition.
\end{abstract}

Mathematics subject classification (1991): 15A45, 15A42, 15A60, 47A30.

Key words and phrases: Positive semidefinite matrices, trace inequalities, Golden-Thompson inequality, $\log$ majorization.

\section{REFERENCES}

[1] T. ANDO, Majorization, doubly stochastic matrices, and comparison of eigenvalues, Linear Algebra Appl. 118 (1989), 163-248.

[2] T. ANDO AND F. HIAI, Log majorization and complementary Golden-Thompson type inequalities, Linear Algebra Appl. 198 (1994), 113-131.

[3] H. ARAKI, On an inequalities of Lieb and Thirring, Lett. Math. Phys. 19 (1990), 167-170.

[4] R. Bhatia, Matrix Analysis, Springer, New York, 1997.

[5] J. C. Bourin, Some inequalities for norms on matrices and operators, Linear Algebra Appl. 292 (1999), 139-154.

[6] D.-W. Chang, A matrix trace inequality for products of Hermitian matrices, J. Math. Anal. Appl. 237 (1999), 721-725.

[7] I. D. CoOpe, On matrix trace inequalities and related topics for products of Hermitian matrices, J. Math. Anal. Appl. 188 (1994), 999-1001.

[8] F. HIAI, Equality cases in matrix norm inequalities of Golden-Thompson type, Linear and Multilinear Algebra 36 (1994), 239-249.

[9] F. HiAI AND D. PETZ, The Golden-Thompson trace inequality is complemented, Linear Algebra Appl. 181 (1993), 153-185.

[10] A. W. Marshall AND I. Olkin, Inequalities: Theory of Majorization and Its Applications, Academic, New York, 1979. 